\title{
Bergsoniana
}

\section{Correspondance Henri Bergson - Adolf Keller (1914-1939)}

Henri Bergson et Adolf Keller

Florent Serina (éd.)

OpenEdition

Journals

Édition électronique

URL : https://journals.openedition.org/bergsoniana/460

DOI : 10.4000/bergsoniana.460

ISSN : 2800-874X

Éditeur

Société des amis de Bergson

Référence électronique

Henri Bergson et Adolf Keller, « Correspondance Henri Bergson - Adolf Keller (1914-1939) »,

Bergsoniana [En ligne], 1 | 2021, mis en ligne le 01 juillet 2021, consulté le 10 novembre 2021. URL: http://journals.openedition.org/bergsoniana/460 ; DOI : https://doi.org/10.4000/bergsoniana.460

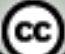

Les contenus de la revue sont mis à disposition selon les termes de la Licence Creative Commons Attribution 4.0 International. 


\title{
CORRESPONDANCE HENRI BERGSON- ADOLF KELLER (1914-1939)
}

\author{
Établie et annotée par Florent SERINA
}

\section{Adolf Keller à Henri Bergson}

Zürich, 8 avril [19]14

Peterhofstatt 6

Monsieur,

Permettez que je vous envoie, avec cette lettre, à titre d'hommage respectueux un petit travail sur votre philoso/phie, qui a d'abord paru dans Wissen und Leben et qui vient d'être publié en brochure par M. Diederichs à Jena. ${ }^{1}$ Je n'aurais pas osé vous soumettre ce petit travail si incomplet si M. Bovet ne m'avait pas dit il y a quelques jours que vous en aviez / parlé avec bienveillance. ${ }^{2}$ J'espère que mon essai contribuera à répandre en Allemagne

1. Keller accompagna son envoi d'une carte typographiée portant l'inscription "Überreicht vom Verfasser » ("De la part de l'auteur »). Nous avons par ailleurs modifié l'orthographe de quelques termes de cette lettre afin d'en fluidifier la lecture.

2. Ernest Bovet (1870-1941), fondateur et directeur de Wissen und Leben (1907-1923), et titulaire de la chaire de littératures française et italienne de l'Université de Zurich (1901-1922). Bovet avait cru opportun de clarifier son rapport au vitalisme bergsonien : "Quelques personnes, qui s'intéressent à mes idées sur la littérature et sur la vie en général, ont supposé chez moi une forte influence de Hegel et de Bergson. Ce point demande une explication très franche. Mes philosophes préférés furent toujours Spinoza, Pascal, Montesquieu et Sully Prudhomme. Je n'ai jamais lu une page de Kant, ni de Hegel, et n'ai lu que tout récemment deux ouvrages de Bergson [...]. [S]'il y a, sur certains points, quelque analogie entre ses idées et les miennes, c'est qu'un courant général nous entraîne 
et en Suisse et dans un public qui ne s'occupe pas professionnellement de philosophie, les idées qui nous ouvrent un chemin nouveau. Dans tous les cas vous voyez qu'il / y a à Zurich et en Suisse en général un groupe de théologiens - car je ne suis pas le seul — qui étudie très intensément vos œuvres et qui attend la suite de votre pensée avec impatience. ${ }^{3}$ Veuillez accepter, Monsieur, l'assurance de ma haute estime et reconnaissance et croire à mes sentiments les plus distingués.

Adolf Keller Pasteur

\section{Henri Bergson à Adolf Keller}

\section{Villa Montmorency \\ 18 av. des Tilleuls Auteuil Paris 9 avril 1914}

Monsieur,

J'aurais dû, depuis longtemps, vous dire avec quel vif intérêt j'ai lu l'étude si pénétrante, si bienveillante aussi, que vous m'avez consacré dans Wissen und Leben et que vous venez de rééditer. Je suis pris, malheureusement, dans un tel / tourbillon d'occupations que je ne dispose pas d'un instant. Aujourd'hui même, je me dispose à partir pour l'Écosse où je dois faire des cours à l'Université d'Édimbourg. ${ }^{4}$ Dès que j'aurais un peu de loisir (mais

vers une nouvelle conception de la vie. Chacun de nous y participe dans la mesure de ses forces et de sa spécialité. Et les 'rencontres' sont aussi frappantes que nombreuses » (Bovet 1911, VII). Bovet précisa aussi dans une note de bas de page d'un article de Keller : "Lorsque j'ai vu récemment M. Bergson à Paris, il m'a exprimé son admiration pour la manière dont $M$. Keller décrivait les principaux traits de sa philosophie dans un espace si restreint, si profondément et si vivante " (Keller 1916, 735 n. 1).

3. Keller évoqua l'existence de ce petit cercle de lecteurs de Bergson qui se réunissait autour de lui, et dont la conception bergsonienne de la psyché aurait facilité la compréhension de l'inconscient freudien (Keller 1953). Peut-être avait-il à l'esprit Hermann Kutter (18631931), Leonhard Ragaz (1868-1945), et Emil Brunner (1889-1966) qui consacra son mémoire de fin d'études de 1913, puis sa thèse d'habilitation de 1915 à Die Bedeutung $H$. Bergson für die Religionsphilosophie (L'Importance d'H. Bergson pour la philosophie religieuse). 4. Bergson donna entre le 21 avril et le 22 mai 1914 une série de conférences dans le cadre des Gifford Lectures, intitulée " The Problem of Personality. » Essentiellement centrées sur la pensée de Plotin — seul "psychologue " de l'Antiquité — celles-ci étaient dans la continuité de ses leçons prononcées cette même année au Collège de France. Une seconde série de conférences avait été programmée, mais elle fut annulée en raison du 
quand cela arrivera-t-il ? je n'ai pas pris une demi-journée de congé depuis des années !) je vous écrirai plus longuement. ${ }^{5}$ Je voudrais / éclaircir une ou deux au moins des difficultés que vous signalez. — Aujourd'hui, je dois me borner à vous envoyer mes très vifs remerciements, et j'y joins l'assurance de mes sentiments distingués et dévoués.

H. Bergson

\section{Henri Bergson à Adolf Keller}

Paris, 31 rue d'Erlanger

21 oct.[obre] 1919

\section{Cher Monsieur,}

Je viens à vous remercier tout de suite d'avoir consacré à mon dernier livre un article de la Gazette de Zürich. ${ }^{6}$ Vous y analysez avec une précision extrême les parties chapitres que vous avez choisis. Ce que vous

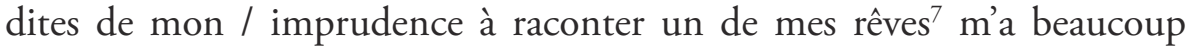
amusé : je suis curieux de savoir ce qu'un psycho-analyste comme vous y découvrirait. $^{8}$

déclenchement de la Première Guerre mondiale. Leur texte qu'il n'a pas voulu publier de son vivant, fut à sa demande détruit avec ses autres manuscrits après sa mort. Seuls des sommaires nous sont parvenus (voir Bergson 1972, 1051-1086).

5. Dans d'autres lettres rédigées quelques jours auparavant, Bergson disait à avoir « dû supprimer toutes vacances » et ne plus savoir "ce que c'est que d'avoir une demi-journée de loisir, même le dimanche " (Bergson 2002, 575) ; ou bien encore être "surchargé de besogne, au point d'avoir à travailler presque jour et nuit " (Bergson 2002, 575).

6. Keller, dans un numéro de la Neue Zürcher Zeitung consacrée à L'Énergie spirituelle (Keller 1919a).

7. Référence au récit onirique où l'aboiement d'un chien se transforme dans le sommeil du philosophe en grondement d'une assemblée, relaté dans sa conférence sur "Le rêve " (1901), parue initialement dans le Bulletin de l'Institut psychologique international, puis avec de légères modifications dans L'Énergie spirituelle. Pour une interprétation historique de ce songe, qui fait écho au rêve de la guillotine d'Alfred Maury (voir Carroy 2012, 296-298).

8. Keller écrit dans cet article : "Dans une étude sur le rêve, Bergson ne fait que montrer à quel point la psychanalyse a creusé plus en profondeur, qui, soit dit en passant, lui est proche. Il est même si imprudent qu'il raconte l'un de ses rêves, ce qui peut apparaître significatif au spécialiste pour ce qui est de la tension intérieure de sa philosophie. » (Keller 1919a, traduction personnelle). L'École de Zurich préférait faire état de matériaux issus de leur pratique clinique plutôt que de rêves issus de leur propre anamnèse. Ainsi en 1911, Jung, qui privilégiait alors l'exposé de rêves de patients, avait cherché à convaincre 
L'autre article de la Gazette, auquel vous faites allusion dans le mot que vous avez bien voulu m'adresser, ne m'est jamais parvenu. Je tiendrais beaucoup à le lire. Pouvez-vous m'en / envoyer un autre exemplaire ? Je vous en remercie d'avance, et je vous prie de croire à mes sentiments bien dévoués.

H. Bergson

\section{Henri Bergson à Adolf Keller}

\section{Paris, 31 rue d'Erlanger}

3 nov[embre] 1919

\section{Cher Monsieur,}

J'apprends avec plaisir que vous allez venir à Paris. Je n'ai pas l'intention de m'absenter pendant ce mois. Si donc vous voulez bien m'avertir du moment de votre arrivée et du temps que vous avez l'intention de rester, je vous proposerai un rendez-vous. Nous continuerons la conversation philosophique dont je conserve un si bon souvenir.

J'ai lu, dans la Gazette de Zurich /que vous m'avez adressée, l'article que vous aviez bien voulu me consacrer, et je vous remercie de la sympathie que vous avez témoignée à mes travaux en répondant aux attaques dirigées contre eux. ${ }^{9}$ Mais j'estime que vous avez fait beaucoup trop d'honneur à

Freud de la nécessité de revoir Die Traumdeutung en fonction d'une méthodologie plus rigoureusement formulée, quitte à entraîner la refonte totale de ce livre que son auteur avait présenté comme un "morceau de $[\mathrm{s}]$ on auto-analyse " (préface à la $2^{\mathrm{e}}$ édition de 1909). Voir la lettre de Jung à Freud du 14 février 1911 (Freud et Jung 1975, 235 J). Ce rêve de Bergson semble d'ailleurs n'avoir jamais fait l'objet d'une analyse rétrospective, que ce soit à Vienne ou à Zurich.

9. Keller (1918a). Cet article avait écrit en réaction à celui qu'avait signé Paul Schweizer (1852-1932), professeur d'histoire à Zurich, à l'encontre du théologien réformé Leonhard Ragaz et de son socialisme religieux : "Unsere Grenzen gegen das Neuland Wolkenkuckucksoder Pfahlbau-Heim, " publié en six parties entre le 23 juin et le 5 juillet 1918 dans le même quotidien. Dans la dernière (intitulée "Zwingli oder Robespierre »), Schweizer lui reprochait d'avoir consacré des conférences à Bergson, alors que ce dernier était accusé d'avoir plagié Schopenhauer, citant notamment le pamphlet d'Hermann Bönke, Plagiator Bergson (1915). Keller écrivit à Ragaz, sans doute peu après sa publication de cet article : "Je crains que vous ne soyez déçu par la nature sommaire de ma défense de Bergson, qui ne voulait pas d'un affrontement dans un quotidien. Tout d'abord, parce que je suis pour ainsi dire épuisé en ce moment. Ensuite, parce que je ne souhaitais pas que par une approche trop pointue, les écrits polémiques de Bönke et Brockdorf puissent se répandre 
l'adversaire — ou plutôt à l'ennemi — en lui répondant. J'ai eu entre les mains un des opuscules auxquels vous faites allusion : il m'a intéressé en me montrant comment on peut porter la minutie, l'esprit de méthode et de système, jusque dans la haine. ${ }^{10} /$

Avec cette méthode, je me chargerais d'établir qu'il n'y a pas une phrase de Leibniz qui n'ait déjà été écrite par Plotin, et que tout l'essentiel de Schopenhauer lui-même se trouve dans le peu que je connais du Bouddhisme. ${ }^{11}$ Ce serait tout aussi probant, et ce serait absurde.

Croyez, je vous prie, à mes sentiments tout dévoués.

H. Bergson

\section{Henri Bergson à Adolf Keller}

Paris, 31 rue d'Erlanger 18 déc.[embre] 1919

Cher Monsieur,

Je suis bien en retard pour vous remercier de votre aimable lettre et de l'article qui l'accompagnait. Ce compte-rendu de l'ouvrage de Curtius m’a vivement intéressé $:^{12}$ Le cas est probablement unique — ou, tout

auprès des lecteurs — ces écrits peuvent être aisément utilisés pour "faire impression » au détriment de Bergson. Il faut admettre qu'il existe bien des parallèles évidents, y compris sur le plan formel. Il ne s'agissait donc cette fois que de brouiller une impression possible sur le public ou de la rendre aussi inoffensive que possible et de laisser la réponse nécessaire à la question à plus tard. Dans le même temps, j'étais heureux de pouvoir exprimer ma désapprobation de l'attaque contre vous à ma manière, ne serait-ce que dans un aparté. » (Keller 1918c, traduction personnelle). À ce sujet, voir aussi François (2005).

10. Sans doute avait-il à l'esprit le brûlot de Bönke, précédemment cité. Il écrit par ailleurs, le 8 octobre 1919, à Émile Borel, qui souhaitait publier la lettre en réponse aux critiques d'Yves Delage sur Le rire: " depuis plusieurs années, je n'ai répondu à aucune des objections qu'on élevait contre telle ou telle de mes vues dans des articles ou dans des livres. Tout mon temps y aurait passé ; - et je me réserve de répondre plus tard, globalement, à l'ensemble" (Bergson 2002, 884-885).

11. Henri Bergson consacrera plusieurs pages au bouddhisme dans les Deux sources de la morale et de la religion. Il postule pour l'essentiel que la mysticité orientale se serait comme arrêtée à mi-chemin, ne parvenant pas à véritablement atteindre la vie divine.

12. Keller 1919b. Ernst Robert Curtius (1886-1956), philologue allemand, spécialiste des littératures romanes, auteur en 1919 de Die literarischen Wegbereiter des neuen Frankreich (Les pionniers littéraires de la nouvelle France); republié dans Curtius (1952, 5-273). Caterina 
au moins, bien rare — d'un Allemand rendant ainsi pleine justice à la France. ${ }^{13}$

J'aurais dû répondre aussi à la question que vous me posiez au sujet d'une préface éventuelle au livre de M. le Professeur Vodoz. ${ }^{14}$ La psychoanalyse / est fort peu cultivée chez nous, et je ne vois personne qui en soit le représentant attitré. ${ }^{15}$ Elle m'intéresse, pour ma part, vivement ; mais je n’ai guère eu occasion de l'approfondir expérimentalement ; ${ }^{16}$ et d'ailleurs,

Zanfi évoque l'hypothèse d'une rencontre entre Bergson, Otto Baensch et Ernst Robert Curtius lors d'un séjour de ces derniers à Paris courant 1909 (Zanfi 2013, 46).

13. Keller écrit notamment : "Ainsi commence une renaissance spirituelle, un développement créatif, un nouveau courage pour agir et collaborer, qui trouve en Bergson une expression spirituelle qui fait autorité. Face aux critiques allemandes à l'encontre de Bergson, comme on l'entend de plus en plus souvent depuis la guerre, il est précieux de tenir compte des paroles qu'un Allemand oppose ici à cette critique : 'Toutes ces réfutations de Bergson cherchent à faire remonter la nouveauté créative à ce qui a déjà existé, elles cherchent à expliquer rétrospectivement l'intuition uniformément développée comme une mosaïque d'éléments qui ont longtemps existé. Il n'y a pas de motif de pensée bergsonienne qu'ils ne trouvent chez un philosophe antérieur' " (Keller, 1919b, 109, traduction personnelle). Voir aussi Curtius (1925).

14. Jules Vodoz (1866-19?) professeur de français au Gymnasium de Zurich, membre de l'Association zurichoise de psychanalyse, puis membre fondateur du Club psychologique de Zurich. Son ouvrage, "Roland, " un symbole, paru en 1920, montre à partir d'un commentaire du poème "Le mariage de Roland " de Victor Hugo que la critique littéraire aurait intérêt à s'approprier la notion d'inconscient, susceptible de renouveler la compréhension de la littérature romantique, en mettant en lumière certains symboles immémoriaux, source d'apaisement et de délivrance. Cet essai paru chez Honoré Champion à Paris, avec une lettre-préface de Georges Duhamel, est considéré comme l'une des toutes premières études de psychanalyse littéraire signée par un non-médecin. Vodoz semble avoir eu la lettre de Bergson en main, comme le suggère la note ajoutée en bas de la lettre stipulant " bitte zurück an Prof. Keller. »

15. La Société Psychanalytique de Paris ne fut inaugurée qu'en novembre 1926, trois ans après la fondation du groupe de L'Évolution psychiatrique. Avant que la mise sur pied de ce premier groupe soit effective, le docteur René Laforgue avait dit son intention de solliciter Bergson pour qu'il figure dans son comité de patronage aux côtés d'Henri Claude, d'Édouard Toulouse, et de Joseph Rogues de Fursac, ainsi que le montre une lettre à Ernest Jones du 28 octobre 1923 (Laforgue 1923).

16. Sur Bergson et la psychanalyse, voir notamment Poirier 2020, 160-166. Bergson ne s'est jamais allongé sur le divan d'un psychanalyste. Sa lettre à Charles Blondel du 11 janvier 1924, en réponse à l'envoi de son essai au vitriol sur La psychanalyse, conduit de plus à nuancer son enthousiasme à l'égard de la doctrine freudienne (Bergson 2002, 1071). Dans ses Entretiens, Jacques Chevalier rapporte toutefois que, le 12 février 1933, Bergson, " avec une suprême finesse et élégance de parole, nous entretient des médications freudiennes très appréciées des malades en ce que le premier article est de les faire parler d'eux-mêmes et de se confesser. 'Je savais bien que c'était là, pour les hommes, le plus 
m'étant interdit à moi-même depuis bien des années d'écrire aucune préface, je ne pourrais maintenant faire une exception sans blesser un grand nombre de personnes auxquelles j'ai déclaré, quand elles voulaient bien me demander une préface, que je m'étais imposé à cet égard une règle absolue. Mais je vais chercher de nouveau, et, si un nom me vient à l'esprit, je vous l'indiquerai.

Je suis très touché de l'aimable / insistance avec laquelle vous me demandez de venir parler à Zürich. ${ }^{17}$ Mais comment faire ? Je suis pris par une foule de besognes ; et d'autre part, depuis bien des mois, je suis si fatigué que j'ai dû réduire le nombre de mes heures de travail. Mille occupations viennent, hélas! me distraire des recherches depuis longtemps entreprises pour donner une suite à L'Évolution créatrice. La vie est courte, le temps passe, et je me demande avec anxiété si j'arriverai à remplir le programme que je m'étais tracé. Voilà mon "état d'âme. " C'est ce qui fait que - malgré la tentation que j'éprouve / de venir causer avec les philosophes de Zurich et de me mettre en rapport avec des pensées que je sais sympathiques à la mienne - je n'arrive pas à me donner les dix ou douze jours de liberté qu'il faudrait pour préparer une ou deux conférences et pour faire ce petit voyage. Ces dix ou douze jours de liberté, je ne me les suis pas donnés depuis des années ; - je ne sais pas ce que c'est que de prendre des vacances. Mais dès que je pourrai venir, je viendrai. - Croyez, je vous prie, cher Monsieur, à mes sentiments dévoués.

H. Bergson

\section{Henri Bergson à Adolf Keller}

Paris, 32 Rue Vital

15 juin 1922

Cher Monsieur,

J'ai en haute estime l'œuvre de Jung, ${ }^{18}$ qui n'est pas seulement intéressante pour le psychologue et le pathologiste, mais encore pour le

grand plaisir, mais on s'en aperçoit chaque jour davantage...' " (Entretiens avec Bergson, op. cit., p. 181).

17. Keller l'invitait vraisemblablement à s'adresser à la Société philosophique de Zurich (Philosophische Gesellschaft Zürich). À son sujet, voir notamment Keller 1921.

18. La lettre originale montre le début d'une lettre raturée, précédant le nom de Jung; lettre qui pourrait être le début d'un «F, " laissant à penser que Bergson aurait un court 
philosophe : c'est là que la psychanalyse a trouvé sa philosophie. ${ }^{19}$ J'aurais donc été heureux et j'aurais considéré comme un honneur d'écrire une préface pour elle. - Malheureusement, nous nous heurtons ici à une question de principe. Depuis bien des années, en effet, je me suis fait une règle de n'écrire aucune préface / (je n’ai fait ne me suis écarté de cette règle que dans un ou deux cas qui ne pouvaient pas créer des précédents, pour des œuvres qui se rattachaient à la guerre). Je m'étais trouvé jadis dans la nécessité de poser ce principe, parce que c'était le seul moyen de me tirer de certaines difficultés. Et maintenant je m’y suis si souvent référé, j'ai si invariablement allégué cette règle quand on voulait bien me demander parfois avec insistance — de préfacer tel ou tel livre, que je ne pourrais faire une exception sans froisser un grand nombre de / personnes. S'il y a un cas où je voudrais pouvoir faire une exception, c'est celui-ci. Mais je n'en vois réellement pas le moyen. Croyez à mon vif regret.

Si vous me faites l'honneur de consacrer une conférence à mes travaux, j'espère qu'elle sera imprimée et que je pourrai ainsi la lire. ${ }^{20}$

Je suis, malheureusement, de plus en plus absorbé par mes occupations, par la nécessité de travailler à fond pour rattraper beaucoup de temps perdu (car j'ai été très fatigué à un certain moment), et je ne sais quand je pourrai réaliser mon / projet de venir faire une conférence à Zurich.

Croyez, je vous prie, cher Monsieur, à mes sentiments tout dévoués.

H. Bergson

\section{Henri Bergson à Adolf Keller}

Paris, 32 Rue Vital

3 Oct.[obre] 1923

instant confondu Freud et Jung. Ce qui ne ferait que confirmer que Bergson connaissait mieux le "père " de la psychanalyse que son ancien collaborateur zurichois. À moins que cette biffure ne révèle une hésitation quant à l'orthographe du nom de Jung, souvent écrit avec un "Y " pour "Yung, " ou plus rarement de «Iung. "

19. Bergson n'emploie plus, comme dans la précédente lettre, le terme de "psychoanalyse " (que Freud préconisait), mais celui de " psychanalyse " (privilégié à l'origine par des auteurs suisses tels qu'Auguste Forel, Ludwig Frank ou C. G. Jung pour soutenir une vision non exclusivement freudienne de cette méthode d'exploration de la psyché inconsciente) dont l'usage avait, dans les pays de langue française, fini par s'imposer.

20. Cette conférence, qui n'est pas connue, n’a pas été publiée. 
Cher Monsieur,

Je tiens à vous dire le plaisir que j'ai eu à lire le joli article que vous venez de consacrer à Durée et Simultanéité. Vous avez extrait du livre l'idée philosophique proprement dite, — la seule qu'on pût exposer sans entrer dans des détails techniques, - et vous l'avez formulée avec une grande précision. ${ }^{21}$ Tous mes remerciements.

La théorie d'Einstein soulève des / questions intéressantes, passionnantes même, qui m'ont absorbé pendant un certain temps. Je reviens maintenant à mes anciennes recherches.

Veuillez, à l'occasion, me rappeler au bon souvenir de M. Seeholzer, ${ }^{22}$ et croyez, je vous prie, à mes sentiments bien dévoués.

H. Bergson

\section{Henri Bergson à Adolf Keller}

Paris, 32

R. Vital

janv[ier] 1929

Cher Monsieur,

Très souffrant dans ces derniers temps, je n'ai pas encore pu vous dire combien j'ai été touché des félicitations que vous avez bien voulu m'adresser à l'occasion du prix Nobel. Mais vous savez suffisamment le prix que j'attache à ce qui vient de vous. Je garde un souvenir bien vivant des entretiens que nous avons eus ensemble, en particulier du dernier, à St. Cergue.

21. Article non identifié.

22. Heinrich Seeholzer (1883-1932), avocat zurichois, auteur notamment en 1919 d'une biographie de Raymond Poincaré. Seeholzer avait déclaré à l'occasion d'une conférence en France courant 1917: « En vérité le Suisse qui visite vos villes [...] est frappé du formidable essor et de la vitalité accrue d'une nation que l'on croyait usée, dégénérée et qui, tout à coup, prouve la vérité de la thèse du philosophe Henri Bergson sur la force créatrice. » Une note renvoyait à l'" exposition magistrale " qu'avait donnée Adolf Keller de la philosophie de Bergson dans Eine Philosophie des Lebens (Seeholzer 1917, 19). Seeholzer participa par ailleurs à la séance commémorative du $4^{\mathrm{c}}$ anniversaire de l'incendie de la Bibliothèque de Louvain, en août 1918, dont la publication fut accompagnée d'une lettre d'Henri Bergson. 
Ma femme, très sensible à votre aimable mot, vous envoie ses remerciements. J'y / joins les miens et je vous prie, cher Monsieur, de croire à mes sentiments tout dévoués.

H. Bergson

\section{Adolf Keller à Henri Bergson}

8 février 1939

Monsieur Bergson

47 Boulevard Beauséjour

$\underline{\text { Paris. }}$

Mon cher Maître,

Beaucoup de temps s'est écoulé depuis que vous m'avez fait l'honneur, en 1918, de m'inviter à votre réception à l'Académie Française et après à votre table. Je vous ai vu depuis lors de nouveau à Paris, et une fois à St. Cergue, mais l'occasion ne s'est plus présentée de vous voir plus souvent, puisque je vous savais malade et que moi-même je fus immergé dans un nouveau travail qui absorbait mes forces presque entièrement. Je parle de ce mouvement œecuménique, auquel je suis affilié. ${ }^{23}$ Cela fait, que la philosophie rentre à l'arrière-plan de mes occupations, ce qui ne signifie pas que je ne m’y intéresse pas et particulièrement à votre œuvre philosophique, qui avait et a encore un intérêt si capital pour moi. J'ai parlé l'autre jour avec M. Charletti ${ }^{24}$ qui, à ma grande joie, m'a appris, que malgré votre maladie, vous ne vivez pas dans une réclusion complète et que vous continuez à vous intéresser aux choses de l'esprit, comme je l'ai vu récemment dans une déclaration que vous avez signée. ${ }^{25}$

Pour ne pas disparaître entièrement de votre mémoire, je tiens donc à me rappeler à vous par ces lignes et à vous assurer de mon attachement et de ma grande vénération au grand maître que vous étiez et que vous êtes encore pour le développement de ma pensée.

23. Référence à son rôle de secrétaire général de Life and Work.

24. Vraisemblablement Sébastien Charléty (1867-1945), historien et haut fonctionnaire français.

25. Adolf Keller fait sans doute référence à la lettre d'Henri Bergson à Daniel Halévy, écrite en hommage à la mémoire de Charles Péguy, publiée dans Le Temps le 26 janvier 1939 ; republié sous le titre «Bergson à D. Halévy " dans Mélanges (Bergson 1972, 1585 1587). 
J'aimerais, lors de mon prochain séjour à Paris vous demander s'il serait possible de vous faire une visite et dans tous les cas, je vous assure, cher Maître, de mes sentiments les plus distingués en vous priant de me rappeler à la mémoire de Madame et Mademoiselle Bergson, que j'ai eu l'honneur de rencontrer à votre table et plus tard à St. Cergue. ${ }^{26}$

[A. Keller]

\section{Adolf Keller à Henri Bergson}

23 Octobre 1939

Monsieur Henri Bergson

Membre de l'Académie Française

47 Boulevard Beau-Séjour

Paris.

Mon cher Maître,

Les journaux nous apprennent que vous célébrez votre $80 \mathrm{me}$ anniversaire. Je l'ai appris seulement avant cette date, et je me suis empressé de rédiger un article pour célébrer cet anniversaire dans un journal suisse. Malheureusement ce journal avait déjà reçu un tel article, de sorte qu'il ne me reste qu'à vous écrire personnellement pour vous présenter mes chaudes félicitations et remerciements. ${ }^{27}$

C'est bien loin depuis que vous m'avez, pendant la dernière guerre, accordé la possibilité d'assister à votre réception à l'Académie Française, et de m'entretenir avec vous à votre table. ${ }^{28}$ Mais cette journée reste gravée dans ma mémoire, et bien qu'un énorme travail au service d'une organisation internationale ne m'a plus permis de m'occuper activement

26. Adolf Keller n'a selon toute vraisemblance pas été reçu par Henri Bergson cette annéelà. Une lettre non datée de Robert de Traz (1884-1951) à ce dernier, datant de septembre ou octobre 1939 indique que Keller ne séjourna qu'un seul jour dans la capitale française. L'essayiste lui conseilla de rendre visite à Wladimir d'Ormesson, journaliste au Figaro, à Louis Gillet, ainsi qu’à Georges Duhamel (Traz (de)).

27. Les Basler Nachrichten auxquels Adolf Keller collabora plusieurs fois, avait publié un hommage, repris par la presse romande, dont le Journal de Genève et la Gazette de Lausanne du 19 octobre 1939, affirmant notamment que depuis René Descartes "la philosophie française n'a pas eu de plus illustre représentant que Bergson. »

28. Voir Keller 1918b. 
de travaux philosophiques, votre pensée m’a accompagné pendant toutes ces années et pendant beaucoup de voyages.

J'étais si heureux d'apprendre que l'état de votre santé vous ai [sic] permis de couronner votre ouvre philosophique sur la morale et la religion, dont nous nous sommes déjà entretenus par anticipation en 1918.

Ce que vous disiez alors à l'Académie Française devant l'élite de France, déjà vers la fin de la guerre, ne nous laissait pas prévoir que nous passerons une seconde fois par les mêmes angoisses.

Veuillez croire, cher Maître, que non seulement votre pensée, mais votre cause a de vrais et de fidèles amis dans d'autres pays, et je vous prie d'accepter dans ce sens avec mes chaudes félicitations, mes voux les plus sincères, et la prière de me rappeler à la mémoire de Mme. Bergson.

[A. Keller]

\section{Bibliographie}

Bergson, Henri. 1972. Mélanges : Lidée de lieu chez Aristote, Durée et simultanéité, correspondance, pièces diverses, documents. Édité par André Robinet. Paris : Presses universitaires de France.

Bergson, Henri. 2002. Correspondances. Édité par André Robinet. Paris : Presses universitaires de France.

Bovet, Ernest. 1911. Lyrisme, Épopée, Drame. Une loi de l'histoire littéraire expliquée par la Révolution générale. Paris : Colin.

Carroy, Jacqueline. 2012. Nuits savantes. Une histoire des rêves (1800-1945). Paris : Éditions de l'EHESS.

Curtius, Ernst Robert. 1925. Französischer Geist im neuen Europa. Stuttgart, Berlin, Leipzig : Deutsche Verlags-Anstalt.

Curtius, Ernst Robert. 1952. Französischer Geistim zwanzigsten Jahrhundert. Berne : Francke.

François, Arnaud. 2005. "Bergson plagiaire de Schopenhauer? Analyse d'une polémique. "Études Germaniques 60(3) : 469-490.

Freud, Sigmund et Carl Gustav Jung. 1975. Correspondance. Paris : Gallimard.

Keller, Adolf. 1916. "Tatsachen und ihre Deutung. "Wissen und Leben $16: 729-736$.

Keller, Adolf. 1918a. "Ist Bergson ein Plagiator ? " Neue Zürcher Zeitung 14 juillet. 
Keller, Adolf. 1918b. "Die Ausnahme Bergsons in die Akademie. " Neue Zürcher Zeitung 3 février.

Keller, Adolf. 1918c. Lettre à Leonhard Ragaz du 14 juillet 1918. Fonds Ragaz. Staatsarchiv des Kanton Zürich.

Keller, Adolf, 1919a. "Ein neues Buch von Bergson. " Neue Zürcher Zeitung 12 octobre.

Keller, Adolf. 1919b. «Zur neuer französischen Geistesgeschichte. » Wissen und Leben, XXII, 3 : 108-114.

Keller, Adolf. 1921. «Eine philosophische Gesellschaft. » Wissen und Leben 16(19) : 924-929.

Keller, Adolf. 1953. Entretien avec K. Eissler. Freud Papers. Library of Congress, Washington D. C.

Laforgue, René. 1923. Lettre à Ernest Jones du 28 octobre 1923. Ernest Jones Collection. Institute of Psychoanalysis. Londres.

Poirier, Jacques. 2020. Les Lettres françaises et la psychanalyse (1900-1945). Dijon : Éditions Universitaires de Dijon.

Seeholzer, Heinrich. 1917. France et Suisse. Discours prononcé à Bordeaux à l'association catholique des étudiants de l'Université le 9 février 1917. Zurich, Paris : Art. Institut Orell Füssli éditeurs, Librairie Fischbacher.

Tranz (de), Robert. Lettre non datée à Afolf Keller. Fonds Keller. Stadtbibliothek de Winterthur.

Zanfi, Caterina. 2013. Bergson et la philosophie allemande 1907-1932. Paris : Armand Colin. 
